\title{
Botella al mar para docentes lectores
}

\author{
Es un motivo gratificante para Nodos y Nudos celebrar, con todos los autores \\ que nos han escrito hasta la fecha, la aparición de la edición número 21 de \\ la revista. Son ya largos los años de historia de la publicación y muchos los
}

maestros que han compartido con sus pares sus experiencias, investigaciones y reflexiones pedagógicas. La revista ha logrado varias indexaciones nacionales e internacionales $y$, aunque el objetivo principal es divulgar la escritura de los maestros, el reconocimiento por entidades como Colciencias constituye una satisfacción para el equipo de la Red de Cualificación de Educadores (RED-CEE), de la Universidad Pedagógica Nacional, así como para los pares académicos, el comité editorial y el equipo editorial.

A muchos nos contaron alguna vez la historia de algún náufrago que, solitario en una isla, escribía un mensaje en un papel, lo metía dentro de una botella, la tapaba herméticamente y la arrojaba al mar, no solo con la esperanza de que alguien la encontrara y leyera el mensaje... ¡sino para que buscara al náufrago y le salvara la vida! Por fortuna, hasta donde recordamos, nunca el mensaje corrió la suerte del náufrago y, al salvarse aquel, se salvó también este.

Cuando un docente escribe un artículo para Nodos y $\mathrm{Nudos}$-y en general cuando se escribe y se publica por medio del papel o en ese mar inmenso llamado Internet-, está reeditando la dramática aventura del náufrago de las historias de infancia: arroja su botella al mar moderno llamado la información, donde espera que alguien lea su mensaje e, idealmente, que busque al autor del mensaje y entable un diálogo con él. Pues no se escribe simplemente para publicar: se escribe sobre todo para comunicarse con personas concretas, tan de carne y hueso como los autores mismos, con una vida concreta y en lugares concretos. Por tanto, leer y ser leído son dos condiciones sine qua non de la escritura. Y antes que ser leído, hay que ser lector.
"Citar es citarse...": con este epígrafe comienza Historias de cronopios y de famas, de Julio Cortázar. Pues bien, parafraseamos una cita del editorialista de la edición número 19 de Nodos y Nudos para recordar que quizás muchos de los docentes que escriben en esta tienen historias para llenar las noches del resto de sus vidas y buscan a quién contárselas. Y esto nos lleva al quid del asunto: nosotros aspiramos a tener siempre maestros que nos escriban, pero sobre todo aspiramos a que haya otros maestros que los lean y que dialoguen con ellos, que es lo que justifica finalmente el esfuerzo de publicar cada número de la revista.

Haciendo una comparación entre la culinaria y la escritura, queremos decir que, si bien el arte de cocinar es un fin en sí mismo, se cocina con el objetivo de que otros disfruten, se recreen y se alimenten de los platos que se preparen. En la misma línea de pensamiento, a los autores que generosamente nos escriben, les pedimos un acto más de generosidad: que se sienten a manteles y "devoren" los artículos de los colegas que escriben en las ediciones anteriores de la Revista, que dialoguen con ellos y que los citen en sus propios escritos. Es la manera de lograr que el mensaje que se arroja al mar de la información caiga en unas manos pródigas. Citarse porque hay una búsqueda común aunque los caminos sean diferentes... Citar a otros articulistas de Nodos y Nudos es, además, una exigencia de las entidades que otorgan la indexación.

Leer los artículos es un primer paso. El otro consiste en comunicarse directamente con sus autores; es una manera de continuar el diálogo y de construir comunidad educativa y de pensamiento. En otras palabras, es la única forma como la botella arrojada al mar de la información 
cumplirá su fin: establecer la comunicación con otro, cercano o lejano. Esa es la finalidad con la cual se publican la dirección electrónica de los autores así como la sección Diredtorio en las páginas finales de cada revista. Y quienes no tengan la colección completa, pueden visitar la página web de Nodos y Nudos en la siguiente dirección: www.pedagogica.edu.co, donde podrán consultar las ediciones anteriores.

Tenemos la sensación de que hay maestros que escriben diferente ahora, pues quizás pesa demasiado el hecho de que esta es una Revista indexada: a veces nos llegan artículos que, más que responder a una necesidad de comunicación, parecen ser hechos "a la medida" de los requisitos de indexación. Respetamos a quienes quieran hacerlo así. Pero también queremos recordarles que la intención original de la revista es contar la vida viva, la vida de carne y hueso de las experiencias, las reflexiones y las investigaciones pedagógicas.

Queremos recordar aquí las palabras del escritor italiano Cesare Pavese, quien en un artículo publicado el 20 de junio de 1945 en el periódico L'Unitá, de Turín, Italia, después recogido en un opúsculo intitulado El oficio de poeta, decía:

Hay un obstáculo al leer -y es siempre el mismo, en cualquier campo de la vida-: la demasiada seguridad en sí mismo (...). Siempre nos hiere el inaudito descubrimiento de que alguien ha visto, no más lejos que nosotros, pero sí de un modo distinto. Estamos hechos de tristes costumbres. Nos gusta asombrarnos, como a los niños, pero no demasiado. (...) Nosotros tenemos ideas, tenemos gustos, ya hemos leído libros: tenemos algo, y como todos los poseedores, tememos por ese algo.

\section{¡Bienvenidos a la edición número 21 de Nodos $y$ Nudos!}

Sea esta la oportunidad de hacer un reconocimiento al trabajo de nuestro colega Daniel Fernando Torres Páez, asistente editorial de Nodos y Nudos durante varios años. Daniel nos dejó como legado su tenacidad y perseverancia en los quehaceres editoriales y en las mil y una labores asociadas: compilar artículos, comunicarse con los autores, asesorar en la escritura a algunos de estos, hacer corrección de estilo, participar en las reuniones editoriales... ¡Que buenos vientos lo acompañen en sus nuevas actividades!

\section{Pares académicos}

Verónica Andrea Catebiel

Especialista en Didáctica de la Química.

Licenciada en Enseñanza de las Ciencias, Universidad Nacional de General San Martín, Argentina. catepol@emtel.net.co

Teresita del Niño Jesús Garduño Doctora en Letras, especialidad Psicopedagogía, Universidad de Neuchâtel, Suiza.

Estudios Superiores en Psicología, Universidad de Neuchâtel, Suiza. Maestría en Ciencias, Especialidad en Educación.

Licenciada en Psicología

Educativa, Universidad Autónoma

Metropolitana. Unidad Xochimilco,

México.

gardunot@prodigy.net.mx

Liliana Lacolla

Doctora en Enseñanza de las

Ciencias, España.

Licenciada en Química, Universidad San Martín. Buenos Aires, Argentina. lilianaele@yahoo.ar

\section{Carmen Alicia Martínez}

Doctora en Enseñanza de las Ciencias.

Maestría en Enseñanza de las Ciencias, Universidad Pedagógica Nacional.

Licenciada en Química.

cadeut@yahoo.com

João Batista Siqueira Harres Doctor en Educación, Pontificia Universidade Católica do Rio Grande do Sul, PUC-RS, Rio Grande do Sul, Brasil.

Especialista en Planeamiento Energético-Ambiental, Municipio Grande do Sul, Brasil.

Maestro en Educación, Pontificia Universidade Católica do Rio Grande do Sul, PUC-RS, Rio.

Graduado en Física, Universidade Federal de Rio Grande do Sul, UFRGS, Rio Grande do Sul, Brasil. jbharres@univates.br

\section{Steiner Valencia Vargas}

Magíster en Docencia de la Física, Universidad Pedagógica Nacional. Especialista en Docencia de las Ciencias.

Licenciado en Biología. steinerv@uni.pedagogica.edu.co

\section{Comité editorial}

Alfredo Ayarza Bastidas

Especialista en Gerencia Integral de Empresas, Universidad del Rosario.

Miembro de la Cámara Colombiana del Libro.

Miembro de Fundalectura. magis07@colnodo.apc.org coopera2@|atino.net.co

Nicolás Buenaventura

Asesor de la Unesco.

Ingeniero Rural, Universidad de Scranton, Estados Unidos.

Pedagogo de la Historia, Instituto de Ciencias Sociales de Moscú. nicobuena@yahoo.com.mx

Clara Inés Chaparro Susa Doctorado en Historia, Lógica y Filosofía de la Ciencia, Universidad Nacional de Educación a Distancia (UNED), Madrid, España. Magistra en Docencia de la Física y licenciada en Física y Química, Universidad Pedagógica Nacional, Bogotá. chaparro@uni.pedagogica.edu.co

\section{Celso Román}

Becario Comisión Fulbright. Master Fine Arts, Sculpture Instituto Pratt de New York. Médico Veterinario, Universidad

Nacional de Colombia.

Maestro en Bellas Artes con especialización en Escultura, Universidad Nacional de Colombia khokhoto@hotmail.com

Juan Carlos Orozco Cruz Magíster en Docencia de la Física, Universidad Pedagógica Nacional, Bogotá, Colombia. orozco@uni.pedagogica.edu.co

Dino de Jesús Segura Robayo Magíster en Educación, Universidad de Nueva York, sede de Búfalo, Estados Unidos.

Físico, Universidad de Leipzig, Alemania.

Docente de excelencia. Premio otorgado por la Alcaldía de Bogotá. apriori@telecom.com.co 